\title{
Wavefront Coherence Area for Predicting Visual Acuity of Post-PRK and Post-PARK Refractive Surgery Patients
}

\author{
Daniel D. Garcia $a^{*}$, Corina van de Pol $b$, Brian A. Barsky $a, c$, Stanley A. Klein $c$

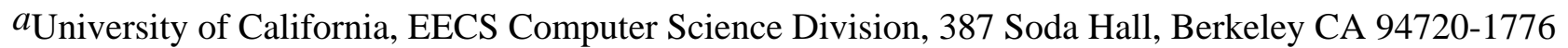

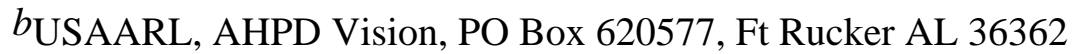 \\ cUniversity of California, School of Optometry, 360 Minor Hall, Berkeley CA 94720-2020
}

\begin{abstract}
Many current corneal topography instruments (called videokeratographs) provide an "acuity index" based on corneal smoothness to analyze expected visual acuity. However, post-refractive surgery patients often exhibit better acuity than is predicted by such indices. One reason for this is that visual acuity may not necessarily be determined by overall corneal smoothness but rather by having some part of the cornea able to focus light coherently onto the fovea.

We present a new method of representing visual acuity by measuring the wavefront aberration, using principles from both ray and wave optics. For each point $\mathrm{P}$ on the cornea, we measure the size of the associated coherence area whose optical path length (OPL), from a reference plane to P's focus, is within a certain tolerance of the OPL for P.

We measured the topographies and vision of 62 eyes of patients who had undergone the corneal refractive surgery procedures of photorefractive keratectomy (PRK) and photorefractive astigmatic keratectomy (PARK). In addition to high contrast visual acuity, our vision tests included low contrast and low luminance to test the contribution of the PRK transition zone. We found our metric for visual acuity to be better than all other metrics at predicting the acuity of low contrast and low luminance. However, high contrast visual acuity was poorly predicted by all of the indices we studied, including our own.

The indices provided by current videokeratographs sometimes fail for corneas whose shape differs from simple ellipsoidal models. This is the case with post-PRK and post-PARK refractive surgery patients. Our alternative representation that displays the coherence area of the wavefront has considerable advantages, and promises to be a better predictor of low contrast and low luminance visual acuity than current shape measures.
\end{abstract}

Keywords: visual acuity, ray tracing, corneal topography, scientific visualization, refractive surgery

\section{INTRODUCTION}

The cornea is the transparent tissue covering the front of the eye, and instruments to measure the cornea's topography are called videokeratographs (VKs). ${ }^{1,2,3,4,5}$ They typically shine rings of light onto the cornea and capture the reflection pattern with a video camera. The instruments use the pattern to reconstruct an internal model of the cornea to compute and display refractive power for the clinician. Many now offer "acuity indices" based on overall corneal smoothness to analyze predicted visual acuity. We find the vision of post-refractive surgery patients to be poorly predicted by these indices. Our goal is to create a new acuity index that would be a better predictor for these patients.

\footnotetext{
${ }^{*}$ Further author information D.D.G. (correspondence): Email: ddgarcia@cs.berkeley.edu; WWW: http://www.cs.berkeley.edu/ ddgarcia/; Telephone: 510-642-9716; Fax: 510-642-5775

C.v.d.P.: Email: MAJ_Corina_VandePol@USAARL-CCMAIL.Army.mil; Telephone: 334-255-6876; Fax: 334-255-6977 B.A.B.: Email: barsky @ cs.berkeley.edu; WWW: http://www.cs.berkeley.edu/ barsky/; Telephone: 510-642-9838; Fax: 510642-5775

S.A.K.: Email: klein@ @pectacle.berkeley.edu; WWW: http://spectacle.berkeley.edu/VSP/SK.html; Telephone: 510-6438670; Fax: 510-643-5109

More information about this research can be found at http://www.cs.berkeley.edu/optical/ and the color images from the poster that accompanied this paper are at http://www.cs.berkeley.edu/optical/SPIE99/
} 


\section{METHODS}

We created a new metric for visual acuity based on the ability of a cornea to focus light coherently to a single point. To analyze real patient data, we recovered the raw ring data from the VK and then reconstructed the cornea as a mathematical curved surface $^{6}$ in a method similar to the one used in our earlier work. ${ }^{7,8}$ The continuous nature of the surface allowed for arbitrary sampling to query both the position and derivative information that is necessary for our calculation. We showered each cornea with approximately 2,800 light rays, in a $2 \mathrm{~mm}$ radius arranged in a crosshatched pattern, as illustrated by Figure 1 below.

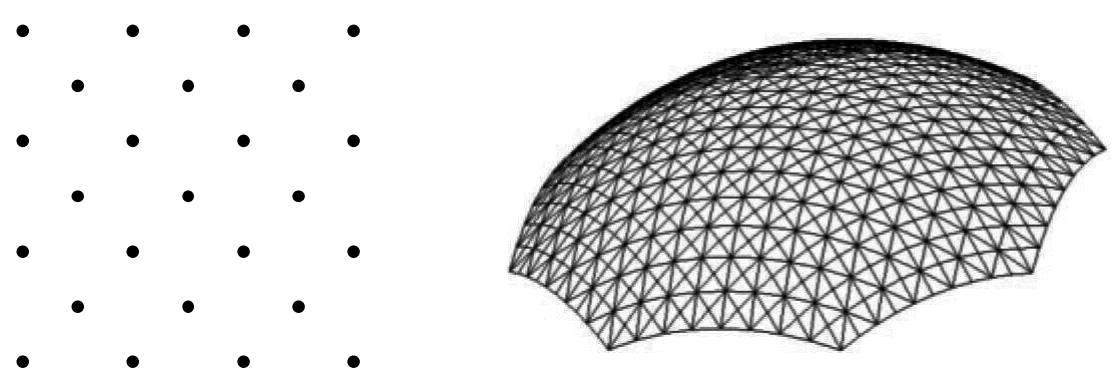

Figure 1: The crosshatched sampling technique on the left was used to query the geometry information from our reconstructed corneal models. The cornea on the right is shown sampled by this method to form a triangular mesh.

Once we had the mathematical machinery in place to analyze an arbitrary recovered corneal surface, all that was needed was to run actual corneal patient data through our system. We used the data from 62 post-refractive surgical corneas and determined the correlation of our acuity index with three measurements of the patient's visual acuity. Then, using the same data, we compared our correlation with that of other popular indices. Finally, we removed two corneas from the sample and analyzed how the fittings changed.

\subsection{Measurements from post-PRK and post-PARK eyes}

The subjects for this study were drawn from patients in the phase III FDA trials of the Nidek excimer laser system. All procedures were performed by one of three refractive surgeons at the Beckman Vision Center, Refractive Surgery Service in the Ophthalmology department of the University of California, San Francisco. The ablation zone diameter of the Nidek laser is 7 millimeters. Photorefractive keratectomy (PRK) involves a single spherical ablation of the anterior cornea. Photorefractive astigmatic keratectomy (PARK) involves a spherical ablation followed by an elliptical ablation to correct for astigmatism. ${ }^{9,10}$ The mean correction for the PRK eyes $(n=31)$ was -7.5 diopters of myopia and for the PARK eyes $(n=31)$ was -5.8 diopters. The PRK patients were $41 \pm 10$ years old and the PARK patients were $48 \pm 10$ years old. Only the latest examination (six or more months postoperative) was used in this study since prior studies have shown that corneal clarity and shape stabilize by this time. ${ }^{11,12}$ The test battery included best spectacle-corrected high contrast visual acuity (HCVA) and small letter contrast sensitivity under standard (SLCT-L) and low luminance (SLCT-D) conditions. ${ }^{13}$ The corneal topography was performed using the TMS-2® corneal topography instrument, ${ }^{14}$ and one investigator (CvdP) completed all visual and topography measurements.

\subsection{Wavefront coherence area}

The idea behind the wavefront coherence area metric is to measure the fraction of the cornea's area inside a specified pupil diameter that is able to focus light coherently onto the retina of the eye. Our model of the cornea is the same one used in our earlier work; ${ }^{15}$ we again ignore the contribution of the lens and consider the cornea to be a uniform material with index of refraction (n) of 1.3375 .

We begin by treating every point on the cornea as a reference corneal point. Next, we determine where the light for that point will focus using Snell's law and Coddington's equations. ${ }^{16,17}$ However, instead of calculating the focus from the mean of the principle refractive curvatures as we did in our earlier work, ${ }^{15}$ here we use the maximum. We will discuss the rationale in the next section when we describe the cylinder correction. From that focus, we first calculate the reference optical path length OPLref through the reference corneal point to a reference plane. The focus is then used to originate a wavefront that we refract back through the cornea and out to a reference plane. We measure the percentage of the wavefront that passes 
through the cornea and $4 \mathrm{~mm}$ diameter pupil whose optical path length (OPL) is within a quarter wavelength of yellow-green light $(550 \mathrm{~nm})$ of the reference OPL. The process is graphically shown in Figure 2. We also include the pseudo-code that implements the calculation of the wavefront coherence area below:

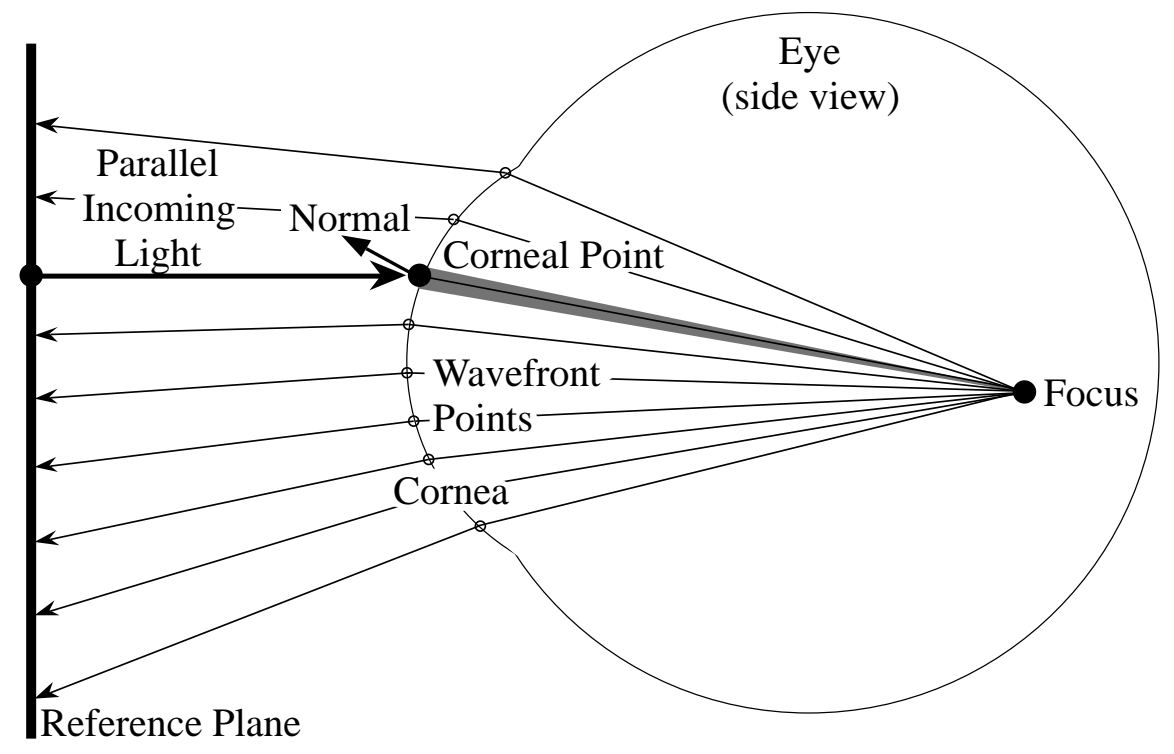

Figure 2: A simple model of a side view of the eye and the technique used for finding the wavefront coherence area.

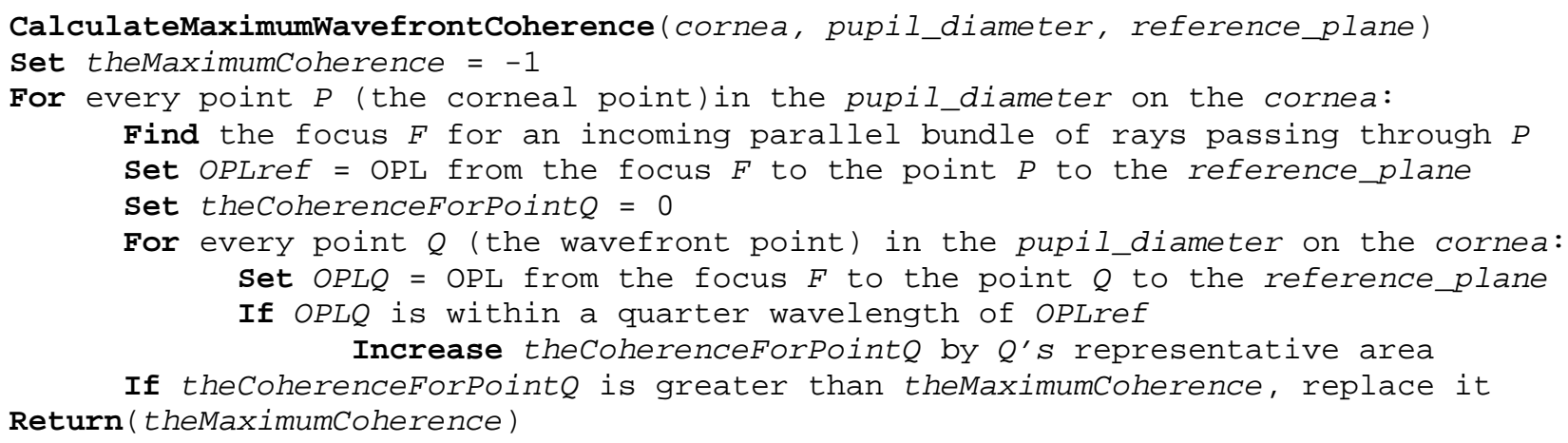

The optical path length is calculated simply by determining the amount of time it would have taken light to travel from the focus to the cornea, through our cylinder corrective lens and to a plane. More specifically, the OPL is the sum of three terms as shown in equation 1 below. The first is the distance, $D_{3 d}$, from the focus to the wavefront point weighted by the index of refraction of the cornea. The second is the refractive cylinder correction, $R C C$, determined from the corneal point and the wavefront point. The third term is the distance, $D_{R P}$, along the refracted exit ray (from the cornea) to the reference plane, which is located at $\mathrm{z}=3 \mathrm{~mm}$, at the entrance pupil plane. If the ray is directed away from the plane, as it will be for most points we consider, this term's contribution is negative. However, for the sake of simplicity, Figure 2 shows all rays directed towards the plane which we have located outside the cornea at a position of approximately $\mathrm{z}=-15 \mathrm{~mm}$.

$$
\mathbf{O P L}=1.3375^{*} D_{3 d}+R C C+D_{R P}
$$

\subsection{OPL refractive cylinder correction}

The second term in the OPL calculation is based on our need to compensate for the dominant refractive cylinder present for most corneal points. Recall that we chose the maximum refractive curvature when determining our focus. That means the bundle of rays around the corneal point in the maximum refractive curvature direction will have very similar OPLs as the reference. We define the "zero refractive cylinder axis" to be the maximum curvature direction; wavefront points along this line will not need any OPL correction. However, due to the non-zero refractive cylinder at the corneal point, wavefront 
points away from the zero refractive cylinder axis will have different OPLs from the reference. We define another term, the "cylinder distance", to be the perpendicular distance from the wavefront point to the zero refractive cylinder axis as shown below in Figure 3.

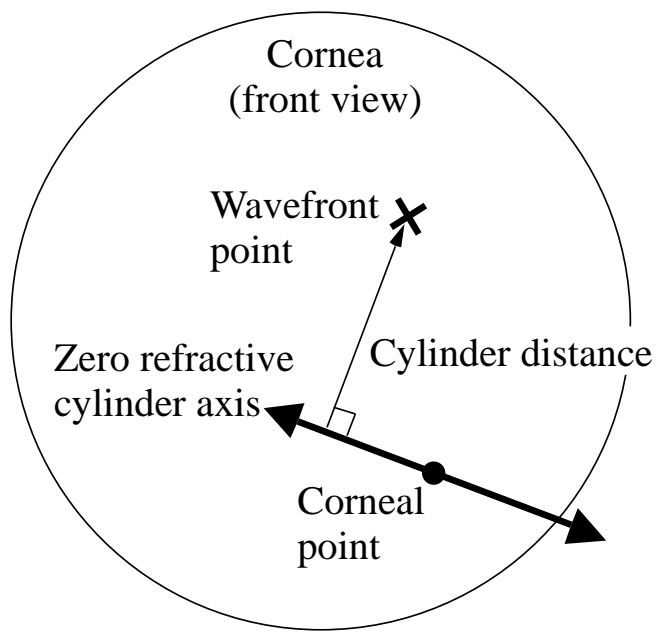

Figure 3: A simple model of the front view of the cornea highlighting the method used to calculate the cylinder distance.

The refractive cylinder correction, $R C C$, is the product of the square of the cylinder distance and half of the refractive cylinder at the corneal point as shown in equation 2 below. This simulates the amount of time a ray would take to travel through a thin cylinder corrective lens with corrective power equal to the refractive cylinder.

$$
R C C=\frac{\text { Cylinder__Distance }^{2} * \text { Refractive_Cylinder_At_Corneal_Point }_{-}}{2}
$$

\subsection{Other visual acuity metrics}

We compared our wavefront coherence area (denoted CA) with three popular metrics taken from the TMS-2® corneal topography instrument: the surface regularity index (SRI), the surface asymmetry index (SAI) and the coefficient of variation of corneal power (CVP). ${ }^{18,19}$ The explanations below are taken directly from the TMS-2® itself:

Surface Regularity Index. The SRI is a correlate to potential visual acuity and is a measure of local fluctuations in central corneal power. When SRI is elevated, the corneal surface ahead of the entrance pupil will be irregular, leading to a reduction in best spectacle-corrected visual acuity. High SRI values are found with dry eyes, contact lens wear, trauma, and penetrating keratoplasty.

Surface Asymmetry Index. The SAI measures the difference in corneal powers at every ring (180 degrees apart) over the entire corneal surface. The SAI is often higher than normal in keratoconus, penetrating keratoplasty, de-centered myopic refractive surgical procedures, trauma, and contact lens warpage. Adequate spectacle correction is often not achieved when SAI is high.

Coefficient of Variation of corneal Power. The CVP is calculated from the Standard Deviation of corneal Powers (SDP) divided by the grand average of corneal powers. This fundamental statistic is high when there is a broad range of powers in the corneal surface and has been found to be a good measure of corneal varifocality. High values of CVP are found in moderate to severe keratoconus corneas as well as corneal transplants in the early post-operative period. Manifest refraction of an eye with high CVP will be difficult to achieve, but attention to refraction is important in such a patient to attain spectacle tolerance. The CVP value given has been scaled up by a factor of 1000 . 


\section{RESULTS}

We charted the correlation of our metric and the other acuity predictors against the actual acuity measurements. We found that none of the predicted indices was able to provide $\mathrm{R}^{2}$ values much above 0.25 , which is what we expected. It is well known that it is difficult to predict the vision of post-refractive surgical corneas. In that context, our predicted acuity metric had the best correlation values for both low contrast (SLCT-L) and low luminance (SLCT-D) vision. It shared the best correlation value with the SAI index for low contrast acuity prediction, but it was the only one that could predict low luminance. HCVA remained poorly predicted by all indices, with correlation values near zero on every chart. The scatter plots for the four acuity predictors are shown in Figure 4, and the correlation summary is in Figure 5.
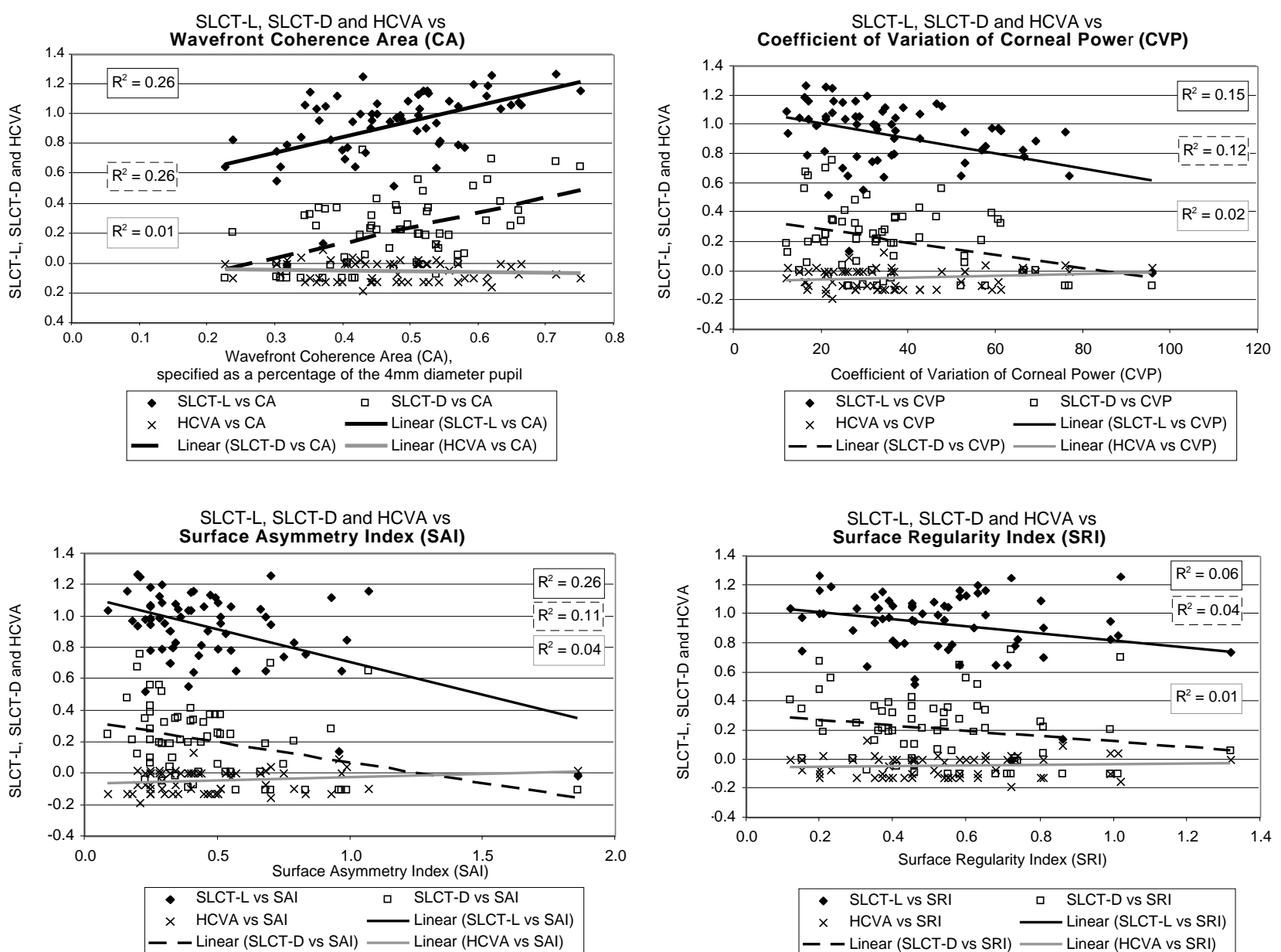

Figure 4: Scatter plots of CA, CVP, SAI and SRI versus the three actual acuity indices, SLCT-L, SLCT-D and HCVA.

\subsection{Analysis of the data fit}

Close analysis of the scatter plots of Figure 4 reveals two patients who have extremely poor low contrast acuity, as measured by the SLCT-L test. These corneas exhibit two of the most de-centered ablations of the group. All of the acuity metrics accurately predict them as having vision far below average, but it is interesting to examine what would happen to the fit of the regression lines if the data from these two corneas were removed from the sample. We would like to know how much of the correlation is based on these two data points.

When these data points are removed, our CA metric is virtually unchanged, as are many of the others. The correlation that changes the most is the SAI vs. SLCT-L comparison. This is especially curious since it was the only correlation whose $\mathrm{R}^{2}$ value was even remotely close to ours with all 62 eyes. Figure 6 shows the comparison of the SAI vs. 
SLCT-L metric before and after removing our two eyes from the sample set. Note that the $\mathrm{R}^{2}$ value drops from 0.26 to 0.03 . This highlights how unstable $\mathrm{R}^{2}$ is for comparing correlation as well as how dependent the SAI metric was on the two poor corneas for achieving its good fit.

Finally, Figure 7 shows the new correlation comparison after removing the two poor corneas from the sample, leaving 60 eyes. Note that with this data, the correlation values for wavefront coherence area remain virtually unchanged and it is now the best predictor of visual acuity for both low contrast and low luminance.

\section{Correlation $\mathbf{R}^{2}$ values for Simulated Indices Compared with Actual Indices for 62 Corneas}

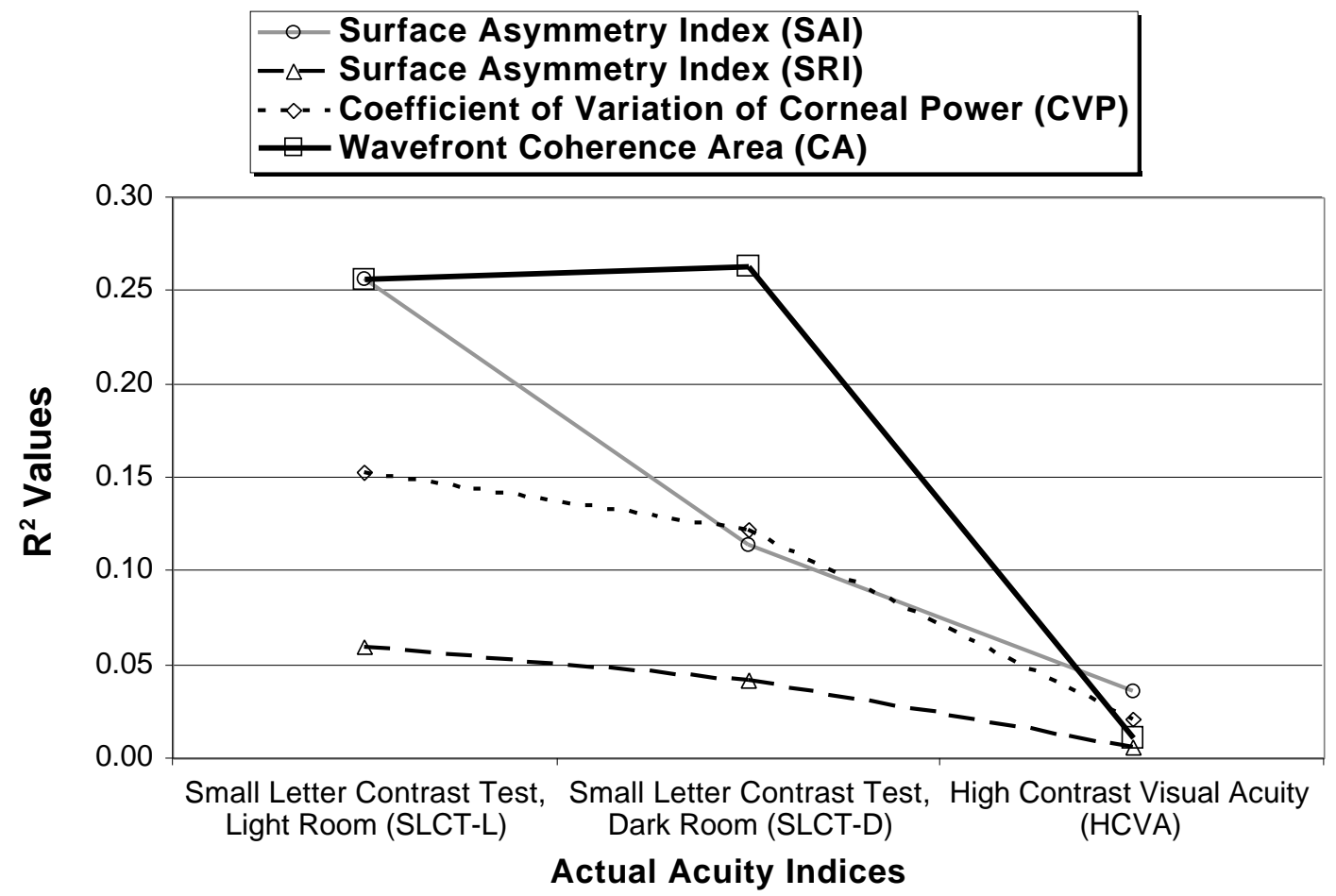

Figure 5: Comparison of correlation $\mathrm{R}^{2}$ values for the four visual acuity predictors.
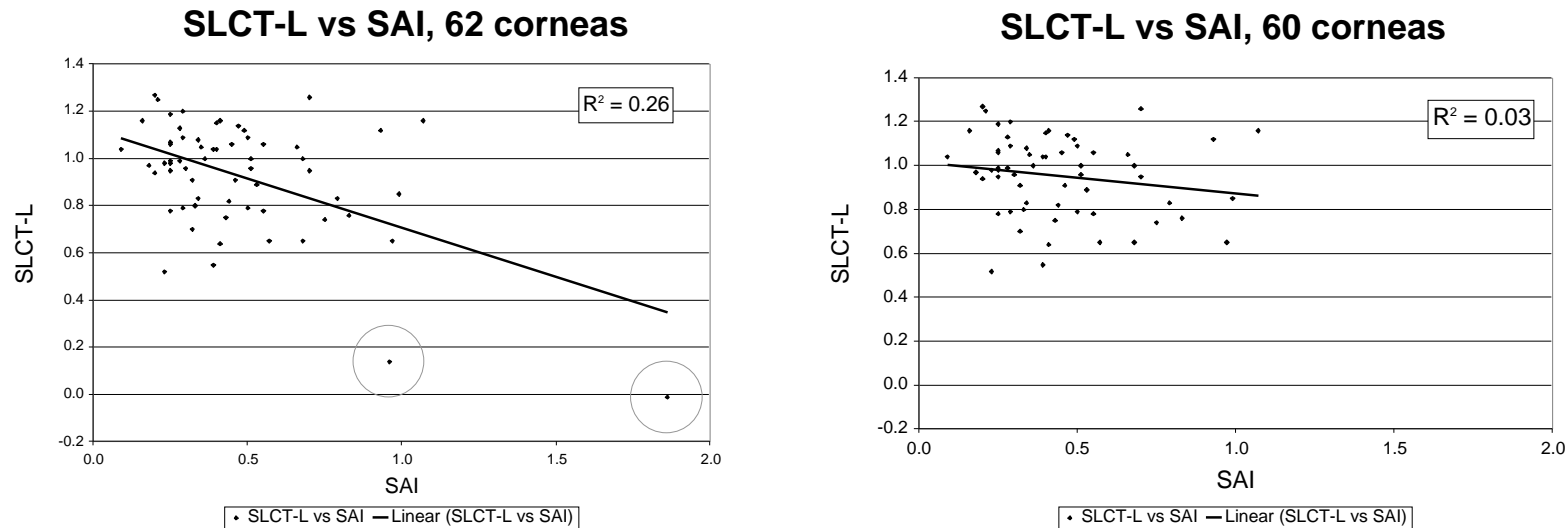

Figure 6: Predictor SAI versus measured low contrast SLCT-L before and after removing the two circled poor performing eyes. 


\section{Correlation $\mathbf{R}^{2}$ values for Simulated Indices Compared with Actual Indices for 60 Corneas}

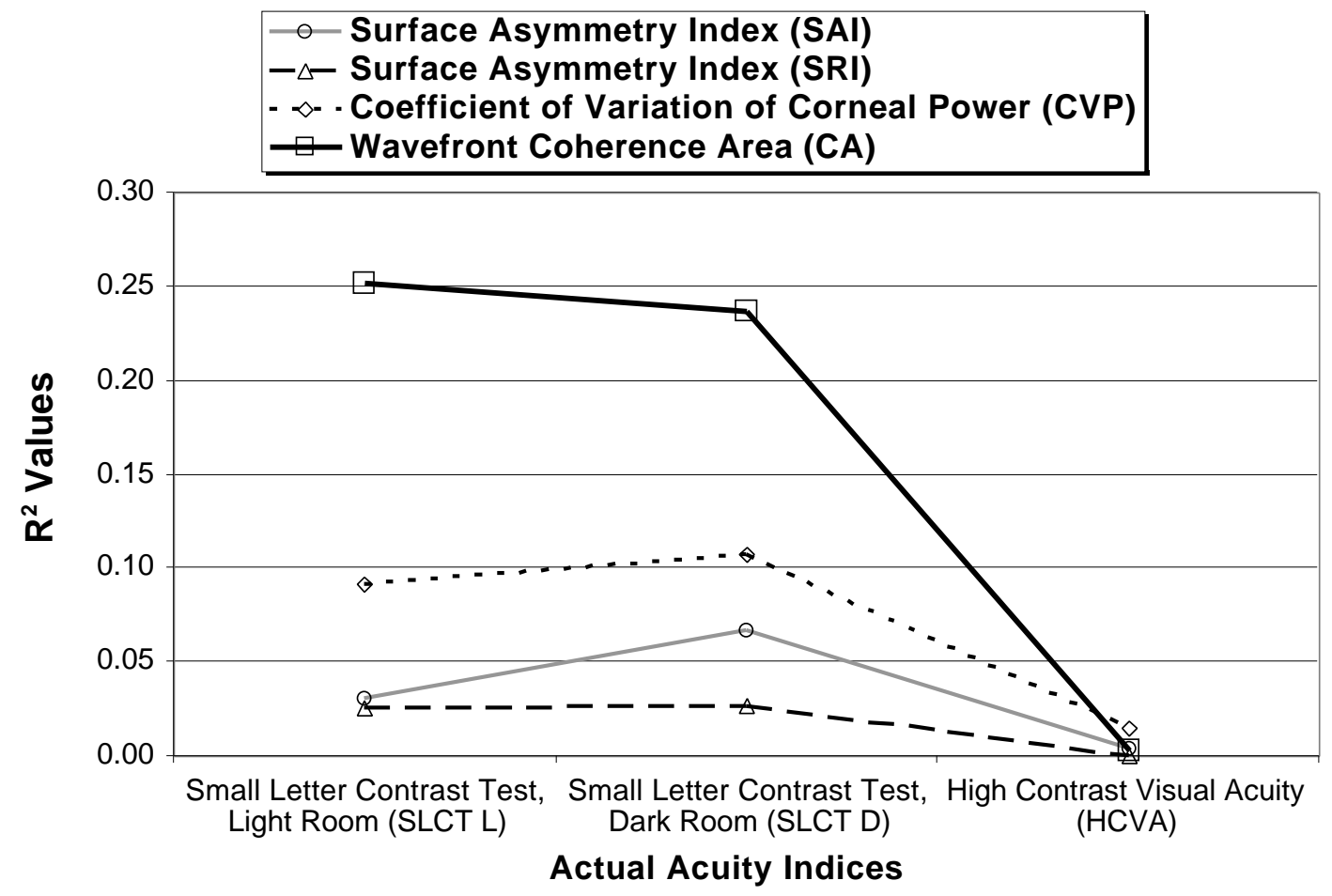

Figure 7: The correlation comparison after removing two patients' corneas from the sample.

\section{CONCLUSION}

We presented a metric for simulating visual acuity based on wave and ray optics called the wavefront coherence area. We evaluated 62 post-PRK and post-PARK corneas and found that our index predicted the low contrast and low luminance acuity for these corneas better than the three popular metrics SAI, SRI and CVP. However, high contrast visual acuity was poorly predicted by all the metrics. Removal of two corneas with extremely de-centered ablations from the sample resulted in a significant decrease in the correlation of the SAI index, with the others remaining relatively constant.

In summary, our representation has considerable advantages, as it more accurately reflects what contributes to visual acuity - the ability of a region of the cornea to focus light coherently onto the retina of the eye.

\section{ACKNOWLEDGEMENTS}

This work was supported in part by the National Science Foundation grant number ASC-9720252, "Visualization and Simulation in Scientific Computing for the Cornea".

\section{REFERENCES}

1. D. S. Klyce, "Computer-assisted corneal topography, high-resolution graphic presentation and analysis of keratoscopy", Ophthalmol. Vis. Sci. 25, pp. 1426-1435, 1984.

2. D. D. Koch, G. N. Foulks, and T. Moran, "The corneal eyesys system: accuracy, analysis and reproducibility of first generation prototype", Refract. Corneal Surg. 5, pp. 424-429, 1989. 
3. R. J. Mammone, M. Gersten, D. J. Gormley, R. S. Koplin, and V. L. Lubkin, “3-D corneal modeling system”, IEEE Trans .Biomedical Eng. 37, pp. 66-73, 1990.

4. J. Wang, D. A. Rice, and D. S. Klyce, “A new reconstruction algorithm for improvement of corneal topographical analysis", Refract. Corneal Surg. 5, pp. 379-387, 1989.

5. S. E. Wilson and D. S. Klyce, “Advances in the analysis of corneal topography”, Surv. Ophthalmol. 35, pp. 269-277, 1991.

6. J. A. Corbin, S. A. Klein, and C. van de Pol. "Measuring Effects of Refractive Surgery On Corneas Using Taylor Series Polynomials", Proceedings of Ophthalmic Technologies IX (this volume), SPIE International Symposium on Biomedical Optics, San Jose, California, January 23-29 1999.

7. M. Halstead, B. A. Barsky, S. A. Klein, and R. B. Mandell, "Reconstructing Curved Surfaces From Specular Reflection Patterns Using Spline Surface Fitting of Normals", Proceedings of ACM/SIGGRAPH '96, New Orleans, 4-9 August 1996, pp. 335-342, 1996.

8. M. Halstead, B. A. Barsky, S. A. Klein, and R. B. Mandell, "A spline surface algorithm for reconstruction of corneal topography from a videokeratograph reflection pattern”, Optom. Vis. Sci. 72, pp. 821-827, 1995.

9. M. J. Goggin, P. F. Kenna, et al. "Photoastigmatic refractive keratectomy for compound myopic astigmatism with a Nidek laser". Journal of Refractive Surgery 13(2): 162-6, 1997.

10. H. Lubatschowski, O. Kermani, et al. "A scanning and rotating slit arF excimer laser delivery system for refractive surgery”. Journal of Refractive Surgery 14(2 Suppl): S186-91, 1998.

11. M. J. Goggin,, P. Kenna, et al. "Haze following photorefractive and photoastigmatic refractive keratectomy with the Nidek EC5000 and the Summit ExciMed UV200". Journal of Cataract and Refractive Surgery 23(1): 50-3., 1997.

12. P. S. Hersh, R. D. Stulting, et al. "Results of phase III excimer laser photorefractive keratectomy for myopia. The Summit PRK Study Group”. Ophthalmology 104(10): 1535-53, 1997.

13. J. Rabin and J. Wicks. "Measuring resolution in the contrast domain: the small letter contrast test". Optom Vis Sci 73(6): 398-403, 1996.

14. D. Brenner. "Modeling the cornea with the topographic modeling system videokeratoscope". Optom Vis Sci 74(11): 8958, 1997.

15. D. Garcia, B. A. Barsky and S. A. Klein, "CWhatUC: A Visual Acuity Simulator", Proceedings of Ophthalmic Technologies VIII, SPIE International Symposium on Biomedical Optics, San Jose, California, January 24-30 1998, pp. 290-298, 1998.

16. O. Stavroudis, "Simpler derivation of the formulas for generalized ray tracing", J. Opt. Soc. Amer. Vol. 66, No. 12, pp. 1330-1333, 1976.

17. J. E. A. Landgrave and J. R. Moya-Cessa, "Generalized Coddington equations in ophthalmic lens design", J. Opt. Soc. Amer. Vol. 12, No. 8, pp. 1637-1644, 1996.

18. S. A. Dingeldein, S. D. Klyce, et al. "Quantitative descriptors of corneal shape derived from computer-assisted analysis of photokeratographs". Refractive and Corneal Surgery 5(6): 372-8, 1989.

19. S. E. Wilson and S. D. Klyce. "Quantitative descriptors of corneal topography. A clinical study". Archives of Ophthalmology 109(3): 349-53, 1991. 